\title{
Gut microbiome of native Arab Kuwaitis
}

\author{
Erica Plummer ${ }^{1}$, Dieter Bulach ${ }^{2,3}$, Glen Carter ${ }^{2}$ and M. John Albert ${ }^{4^{*}}$
}

\begin{abstract}
Background: The human gut microbiome has an important role in health and disease. There is extensive geographical variation in the composition of the gut microbiome, however, little is known about the gut microbiome composition of people from the Arabian Peninsula. In this study, we describe the gut microbiome of Arab Kuwaitis. The gut microbiome of 25 native adult Arab Kuwaitis was characterised using $16 \mathrm{~S}$ rRNA gene sequencing of the V3-V4 regions. Sequencing data were analysed using DADA2. Phylogeny analysis was performed using amplicon sequence variants (ASVs) assigned to the Bacteroides genus and 165 rRNA sequences of Bacteroides type strains to understand the relationships among Bacteroides ASVs.
\end{abstract}

Results: About $63 \%$ of participants were overweight/obese reflecting normal Kuwaiti population. Firmicutes and Bacteroidetes were the dominant phyla detected in the gut microbiome (representing $48 \%$ and $46 \%$ of total sequencing reads respectively). At the genus level, Bacteroides was the most abundant genus in 22 of 25 participants. A total of 223 ASVs were assigned to the Bacteroides genus, eleven of which were present in $50 \%$ or more of study participants, reflecting a high diversity of this genus. Phylogenetic analysis revealed that the Bacteroides dorei/vulgatus group was the most abundant phylogenetic group (representing $11.91 \%$ of all sequence reads) and was detected in all 25 individuals.

Conclusions: Bacteroides was the most abundant genus in the gut microbiome of native Arab Kuwaiti adults, with Bacteroides dorei/vulgatus forming the predominant phylogenetic group. The microbiome composition would also have been influenced by the nutritional status of participants.

Keywords: Gut microbiome, Arab Kuwaiti, $16 \mathrm{~S}$ rRNA gene, Bacteroides, Diet

\section{Introduction}

The human gastrointestinal tract is home to trillions of bacteria [1]. Gut microbes play an important role in digestion of nutrients [2], metabolism of drugs and other compounds [3, 4], and synthesis of micronutrients [5], neurotransmitters and other metabolites [6,7], as well as in the development of gut-specific immune system [8]. The gut microbiome also has an important role in maintaining health, and dysbiosis of the gut microbiome has been associated with various health disorders including metabolic syndrome, diabetes mellitus, obesity, inflammatory bowel disease, colorectal cancer, rheumatoid

${ }^{*}$ Correspondence: john@hsc.edu.kw

${ }^{4}$ Department of Microbiology, Faculty of Medicine, Kuwait University, Jabriya, Kuwait

Full list of author information is available at the end of the article arthritis, atopy, eczema, autoimmune diseases and psychiatric disorders $[9,10]$.

There is a high degree of variation in the gut microbiome composition between individuals and within individuals over time, and people living in different geographical regions tend to have different gut microbiome [11, 12]. The diversity and composition of the gut microbiome is influenced by a variety of different factors including diet, environment, antibiotic use, proximity of hosts and host genetics $[13,14]$ and it is thought that globally, the gut microbiome of healthy individuals segregates into two or three distinct enterotypes based on the abundance of key bacteria-Bacteroides, Prevotella and members of the Clostridiales order $[14,15]$.

Characterising the 'normal' gut microbiome in different geographical regions provides a comprehensive picture of the microbiome from a global perspective. This 
is an important baseline information for understanding dysbiosis of the gut microbiome and how it relates to different populations. There are numerous studies on the gut microbiome of populations from various parts of the world [12, 16-22], but only two studies from the Arabian Peninsula [23, 24], a major geographical region. These two studies are from Saudi Arabia. There are no studies from Kuwait, another country in the Arabian Peninsula. There is genetic variation [25] and variation in food consumption patterns across the Arabian Peninsula [26], which would influence the gut microbiome. Therefore, we studied the gut microbiome of native adult Arab Kuwaitis.

\section{Methods}

\section{Participants}

Healthy volunteers, 18 years or older, born and brought up in Kuwait, and of native Arab Kuwaiti ethnicity were recruited for the study. People with a history of intake of probiotics or antibiotics for the past six months, symptoms pertaining to gastrointestinal tract, hypertension, atopic disease, mental illness and high-risk sexual behaviour were excluded.

Body mass index (BMI) was calculated for participants, and was categorized according to the World Health Organization standard categories: underweight $<18.5$; normal weight 18.5-24.9; overweight 25.0-29.9; obese $\geq 30$ [27].

Participants were presented with a description of a typical Kuwait diet and asked whether their dietary habits agreed with the typical diet or not. Briefly, the Kuwaiti diet consists of meats, dairy products, grains, legumes, vegetables (including leafy greens and herbs), fruits and nuts. A food frequency questionnaire estimated the average daily servings for Kuwaiti adults as 1.9 servings of meat, 3.4 servings of dairy products, 5.3 servings of cereals/cereal products, 3.2 servings of vegetables and 2.8 servings of fruits [28].

\section{Sample collection and laboratory methods}

Participants were asked to provide a fresh faecal sample for microbiome analysis. Faecal specimens were collected by participants into sterile stool containers and transported in a cold box with ice-packs to the laboratory where they were frozen at $-80{ }^{\circ} \mathrm{C}$ within $2 \mathrm{~h}$ of collection and stored until further analysis. DNA was extracted from stored faecal samples using the QIAamp Fast DNA Stool mini kit as previously described [29]. Dried DNA samples were shipped at ambient temperature to Melbourne, Australia for further analysis. PCR amplification of the variable region V3-V4 of the $16 \mathrm{~S}$ rRNA gene (341F/805R) [30] was performed followed by amplicon sequencing on the Illumina MiSeq platform
(Microbiological Diagnostic Unit, The Peter Doherty Institute for Infection and Immunity, The University of Melbourne; and Micromon, Monash University, Victoria, Australia). A blank negative control was processed and sequenced in the same manner described above to allow for identification of reagent contaminants.

\section{Sequence and data analysis}

Primers were trimmed from demultiplexed reads using TagCleaner [31] and the trimmed sequences were processed using the DADA2 pipeline, version 1.6.0 [32]. Quality control, error rate learning and inference of amplicon sequence variants (ASVs) were performed separately for each of the two sequencing runs to account for run-specific error profiles. Following merging of forward and reverse reads, the two runs were merged into a single sequence table. Chimeras were removed from the merged sequence table and taxonomy was assigned using the DADA2 implementation of the RDP Naive Bayesian Classifier and the Silva reference database (v128). Species level assignment was performed using exact matching in the DADA2 pipeline, allowing for multiple matches per ASV. The sequence table was filtered for contaminants identified in the negative control sample and ASVs were removed if they were present at a relative abundance of $0.001 \%$ or less (i.e. fewer than 96 reads).

QIIME 1.9.0 was used to generate rarefaction plots and to determine the core microbiome of specimens, which in this study was defined as ASVs present in at least 85\% of specimens. A heatmap was generated by hierarchical clustering of Bray-Curtis dissimilarity distances with Ward's linkage using the vegan [33] and gplots [34] packages and R v3.4.3 using R studio. Alpha and beta diversity metrics were calculated at ASV level using the vegan package [33]; alpha diversity was calculated using the Shannon diversity index and beta diversity was calculated using the Bray-Curtis index.

The Wilcoxon rank-sum test was used to assess differences in Shannon diversity between microbiome groups as well as assess differences in the Firmicutes: Bacteroidetes ratio of the gut microbiome between individuals with normal BMI compared to individuals with overweight or obese BMI. Statistical analyses were performed using Stata/IC (Version 14.2, StataCorp LP, College Station, USA).

Information relating to the genus Bacteroides and the type strains for each of the species, including accession numbers for the 16S rRNA gene, was obtained from bacterio.net (List of Prokaryotic names with Standing in Nomenclature [35]). Reference sequences were trimmed to include only the V3-V4 region of the 16S rRNA gene prior to the production of the multiple sequence alignment of all Bacteroides ASVs (i.e. including those 
present prior to filtering of the ASV table) and type strain sequences. Multiple sequence alignment and inference of the distance tree was performed using Clustal Omega [36]. The tree was used to group related ASVs and classify these phylogeny groups with names based on the type strain taxa included in the group.

The sequence data from this study were deposited in the NCBI Sequence Read Archive (SRA) under project accession number, PRJNA554702.

\section{Results}

Twenty-five participants (10 males and 15 females, Table 1), aged 24 to 57 years were recruited from March 2017 to May 2017 (Table 1). The body mass index (BMI) value was not available for one participant. Ten (41.6\%) and five $(20.8 \%)$ of the remaining 24 participants were overweight and obese respectively. One participant was diagnosed with Crohn's disease post specimen collection and informed the study team of the diagnosis, but did not report gastrointestinal symptoms at the time of specimen collection. All participants reported consuming a typical Kuwaiti diet.

A total of 9,420,376 $2 \times 300$ base pair reads were generated from sequencing. Following filtering of the ASV table, 9,360,113 sequences (median of 385,526 reads per sample [interquartile range $(\mathrm{IQR})=128,030-537,590]$ ) representing 1621 ASVs were included in the final analysis. Sequencing data were not rarefied as the rarefaction curves (Additional file 1: Figure S1) which confirmed that specimens had been adequately sampled.

Eleven bacterial phyla were detected (Fig. 1). The dominant phyla were Firmicutes and Bacteroidetes (representing $48 \%$ and $46 \%$ of total sequencing reads respectively), and Bacteroidetes was the most abundant phylum detected in the majority of participants $(n=18 / 25,72 \%)$. Firmicutes was the most abundant phylum in seven other participants. Interestingly, participants with a gut microbiome abundant in Bacteroidetes had a lower bacterial diversity compared to participants with a microbiome abundant in Firmicutes (median Shannon diversity index in Bacteroidetes abundant gut $=3.80[\mathrm{IQR}=3.36-3.95]$

Table 1 Participant characteristics

\begin{tabular}{|c|c|c|c|c|c|c|}
\hline Unique ID & Age & Gender & BMI & BMI category & $\begin{array}{l}\text { Shannon diversity } \\
\text { index }\end{array}$ & $\begin{array}{l}\text { Ratio of Firmicutes } \\
\text { to Bacteroidetes }\end{array}$ \\
\hline KW16S_01 & 24 & $\mathrm{~F}$ & 20.6 & Normal & 3.43 & 0.74 \\
\hline KW16S_02 & 25 & $\mathrm{~F}$ & 23.2 & Normal & 3.54 & 0.83 \\
\hline KW16S_03 & 28 & $M$ & 27 & Overweight & 3.82 & 0.78 \\
\hline KW16S_04 & 44 & $\mathrm{~F}$ & 28.3 & Overweight & 3.22 & 0.51 \\
\hline KW16S_05 & 45 & $\mathrm{~F}$ & 21.8 & Normal & 3.39 & 0.66 \\
\hline KW16S_06 & 41 & $M$ & 25.7 & Overweight & 4.75 & 1.86 \\
\hline KW16S_07 & 35 & M & 29.4 & Overweight & 2.93 & 0.29 \\
\hline KW16S_08 & 57 & $F$ & & & 3.04 & 0.72 \\
\hline KW16S_09 & 37 & $M$ & 29.1 & Overweight & 3.93 & 0.57 \\
\hline KW16S_10 & 41 & $\mathrm{~F}$ & 25.9 & Overweight & 4.58 & 1.27 \\
\hline KW16S_11 & 27 & $\mathrm{~F}$ & 18.6 & Normal & 5.06 & 2.93 \\
\hline KW16S_12 & 44 & $M$ & 22.6 & Normal & 4.10 & 0.98 \\
\hline KW16S_13 & 30 & $M$ & 30 & Obese & 4.54 & 2.11 \\
\hline KW16S_14 & 31 & $M$ & 18.7 & Normal & 3.78 & 0.61 \\
\hline KW16S_15 & 26 & $\mathrm{~F}$ & 38.9 & Obese & 3.86 & 0.81 \\
\hline KW16S_16 & 39 & $\mathrm{~F}$ & 41.1 & Obese & 3.96 & 0.55 \\
\hline KW16S_17 & 31 & $\mathrm{~F}$ & 28.1 & Overweight & 4.03 & 0.81 \\
\hline KW16S_18 & 36 & $\mathrm{~F}$ & 21.1 & Normal & 3.36 & 0.72 \\
\hline KW16S_19 & 32 & M & 41 & Obese & 3.44 & 1.35 \\
\hline KW16S_20 & 43 & $F$ & 25.3 & Overweight & 4.04 & 0.83 \\
\hline KW16S_21 & 40 & $\mathrm{~F}$ & 28.5 & Overweight & 3.93 & 0.61 \\
\hline KW16S_22 & 42 & $M$ & 26.1 & Overweight & 3.00 & 0.56 \\
\hline KW16S_23 & 38 & $\mathrm{~F}$ & 23.4 & Normal & 4.34 & 1.47 \\
\hline KW16S_24 & 52 & $M$ & 23 & Normal & 4.83 & 2.75 \\
\hline KW16S_25 & 24 & $F$ & 41.6 & Obese & 3.95 & 0.54 \\
\hline
\end{tabular}

$B M I$ body mass index

a BMI value missing for this participant 


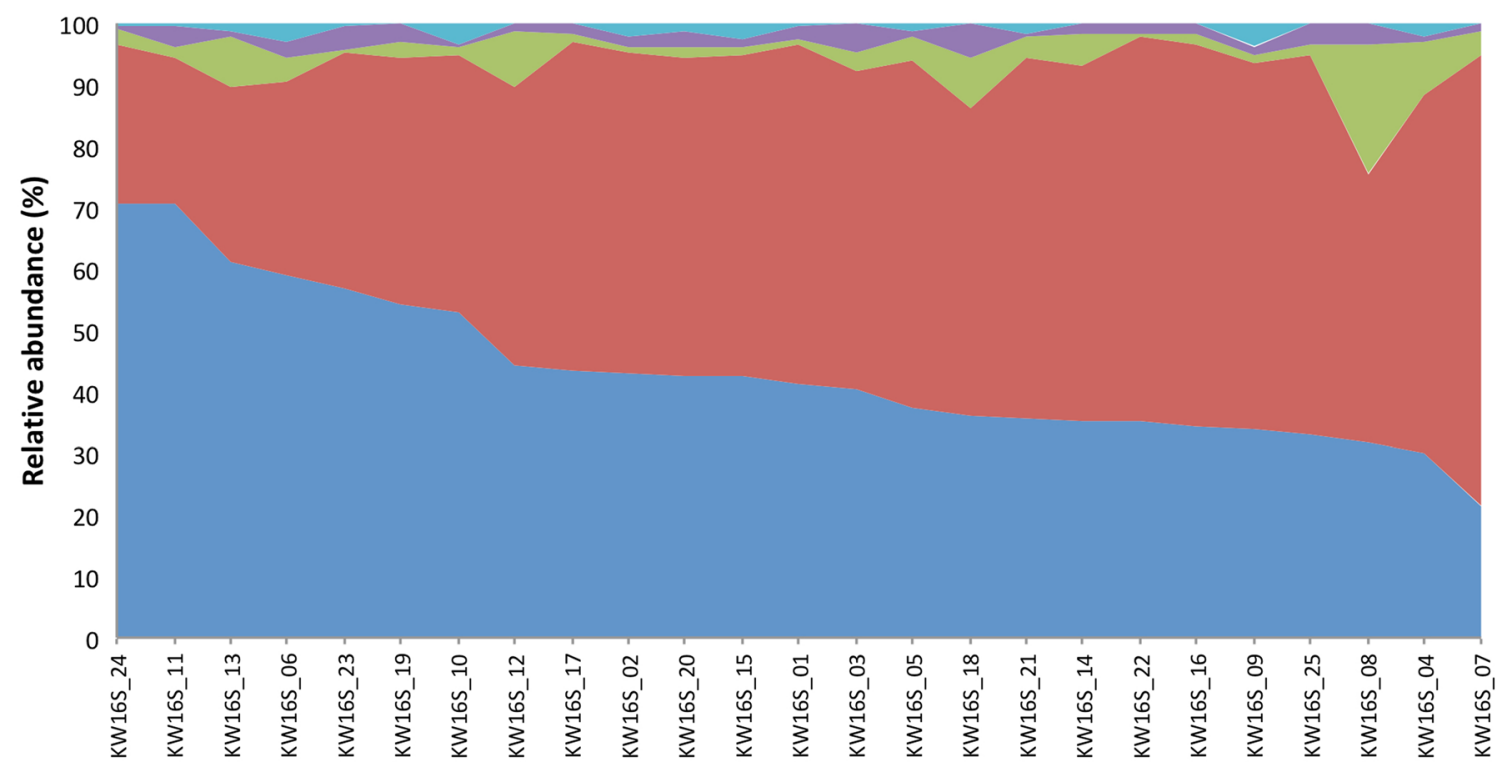

Firmicutes Bacteroidetes Proteobacteria $\square$ Actinobacteria Other

Fig. 1 Relative abundance of the gut microbiome at the phylum level. Area chart shows the gut microbiome profiles of study participants

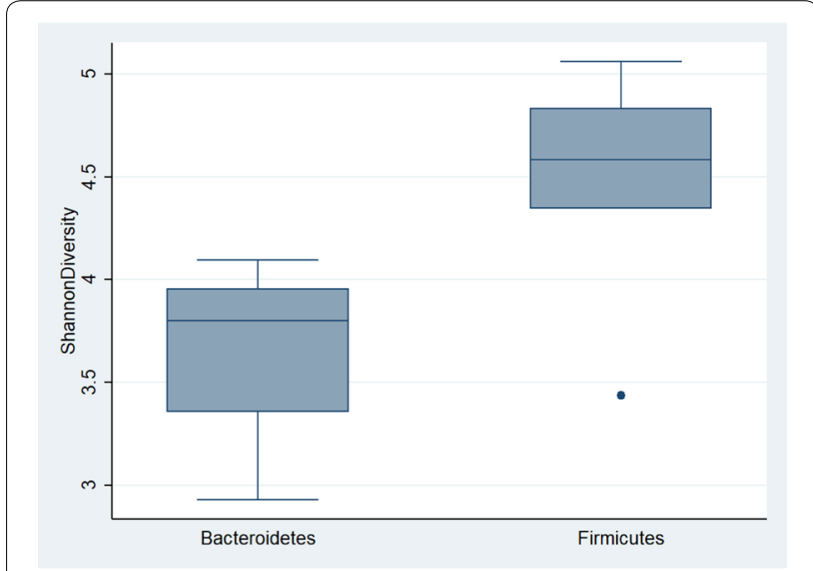

Fig. 2 Relationship between dominant phyla and bacterial diversity. Individuals with a Firmicutes dominated gut microbiome had a higher bacterial diversity (as measured by the Shannon diversity index) compared to individuals with a Bacteroidetes dominated microbiome (Wilcoxon rank-sum test statistic $=-3.15, P=0.002$ )

vs. Firmicutes abundant $=4.58 \quad[\mathrm{IQR}=4.34-4.83]$; $\mathrm{Z}=-3.15, \mathrm{P}=0.002$; Fig. 2).

At the genus level, specimens clustered into two distinct groups driven by the abundance of Bacteroides (Fig. 3), with specimens high in Bacteroides clustering together and specimens with lower Bacteroides abundance clustering together. Bacteroides was detected in all specimens and was the most abundant genus in the majority of participants $(\mathrm{n}=22 / 25,88 \%$, median relative abundance of $37 \%$ [IQR $20-41 \%$ ]). Of the remaining three participants, one had a gut microbiome dominated by Prevotella_9 (52\% relative abundance), one had a highly diverse microbiome with Prevotella_9 being the most abundant genus (10\% relative abundance) and the third had a high abundance of Alistipes relative to other participants $(23 \%$ vs. median abundance of $4 \%$ [range $0-13 \%])$. Interestingly, this participant was the individual diagnosed with Crohn's disease.

The core gut microbiome of participants was represented by 18 ASVs (Additional file 2: Table S1). Fifteen ASVs representing the core gut microbiome were from the Clostridiales order, primarily the Lachnospiraceae and Ruminococcaceae families. ASVs representing Bacteroides fragilis/xylanisolvens (ASV46), Bifidobacterium longum (ASV50) and Escherichia/Shigella (ASV53) were also identified as members of the core microbiome.

Bacteroides ASVs comprised $30 \%$ of all sequence reads. Prior to filtering of the ASV table we identified a total of 223 ASVs assigned to the Bacteroides genus (113 remained post filtering of the ASV table), eleven of which were present in $50 \%$ or more of study participants, indicating a high diversity in Bacteroides spp. across individuals. Twenty-three of the 223 identified Bacteroides ASVs had an average relative abundance greater than $0.5 \%$ and represented more than $80 \%$ of the relative abundance of all the Bacteroides ASVs (Additional file 3: Table S2). Given the 


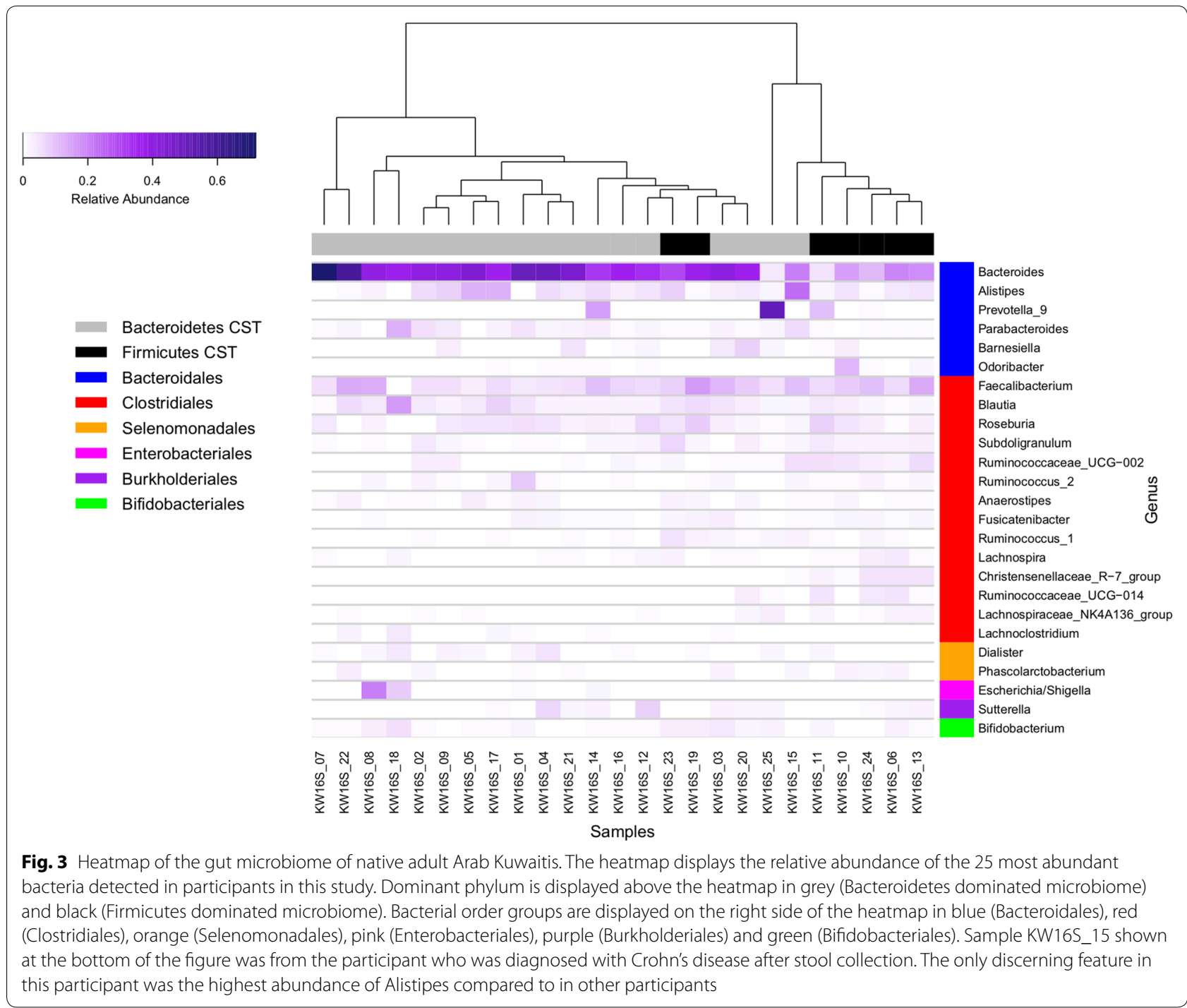

importance of Bacteroides spp. in the gut microbiome and the high diversity within this genus between individuals, we investigated this genus further. We produced a phylogeny from a multiple alignment of the 223 Bacteroides ASVs and published 16S rRNA gene sequence for each type strain in the Bacteroides genus (Additional file 4: Figure S2). The relationships shown in the tree were used to group related ASVs and classify them into phylogeny groups based on the type strain present in the group. Based on the tree, ASV46Bacteroides fragilis/xylanisolvens (which is a part of the core gut microbiome) was more closely related to $B$. xylanisolvens/acidofaciens/caecimuris type strains than B. fragilis type strain. The Bacteroides dorei/vulgatus group was the most abundant phylogeny group (representing $11.91 \%$ of all sequence reads) and was detected in all samples, whereas the Bacteroides fragilis group represented $0.73 \%$ of total reads and was detected in only 12 of the 25 samples (Additional file 5: Table S3).

Information about BMI was available for 24 participants; nine participants had a BMI of normal, ten had a BMI of overweight and five were considered obese (Table 1). There was no observed difference in the ratio of Firmicutes: Bacteroidetes between individuals with a normal BMI and overweight or obese individuals (median Firmicutes: Bacteroidetes ratio in normal $\mathrm{BMI}=0.83[\mathrm{IQR}=0.72-1.47]$ vs. overweight/ obese $=0.78[\mathrm{IQR}=0.55-1.27] ; \mathrm{P}=0.1440$ ). 


\section{Discussion}

It is well established that Firmicutes and Bacteroidetes typically dominate the gut microbiome of healthy adults across the world [37]. This is consistent with our finding that the gut microbiome of adult native Arab Kuwaitis is dominated by either Firmicutes or Bacteroidetes. Individuals with a Firmicutes dominated gut had an increased bacterial diversity compared to those with a Bacteroidetes dominated gut, and Bacteroides was the most abundant genus in all but three individuals.

The limited data describing the gut microbiome of people in the Arabian Peninsula has focused on individuals from Saudi Arabia [23, 24], and describes the dominant phyla to be Firmicutes and Actinobacteria. Diet is thought to be a major driver of differences in the gut microbiome composition seen globally $[14,38]$, and the high abundance of Bacteroidetes in our cohort compared to previous studies of Arabian Peninsula inhabitants could be a result of differences in diet between Kuwaitis and Saudi Arabians. The Kuwaiti diet is generally considered a blend of Arabian, Persian, Indian and Mediterranean cuisines. However, Arabian and Western fast foods rich in meat, sugar and fat are becoming increasingly popular with a consequent rise in the prevalence of obesity, diabetes and hypertension [39]. Bacteroides abundance has been associated with Westernized diets high in animal fat and protein [14], and a previous study demonstrated that introduction of a four-day animal based diet resulted in decreased abundance of Firmicutes in the gut microbiome and a subsequent increase in the abundance of spp. from the Bacteroides, Alistipes and Bilophilia genera [40]. It is possible that the high relative abundance of Bacteroidetes observed in our cohort is due to the increasing popularity of Westernized diets among Kuwaitis; however larger studies with more detailed dietary information are needed to investigate this further. It is worth comparing the Kuwaiti flora with the floras in low- and middle-income countries. The remote huntergatherer populations such as the Hadza from Tanzania, Pygmies from Central Africa, Mases from Peru and Amerindians from Venezuela have a high microbial diversity and an enriched taxa consisting of Prevotella, Succinovibrio, Treponema, Cyanobacteria, Tenericutes, Clostridium, Catenibacterium, Eubacterium, Lachnospira and Salmonella. Prevotella is thought to enhance the ability to digest and extract valuable nutrition from fibrous plant foods. People living in traditional farming or fishing communities like the Bantus of Africa, the Tunapuco population of the Andean highlands or the rural Malawian communities have a high microbial diversity and an enriched taxa consisting of Prevotella, Succinovibrio, Treponema, Ruminococcus and Bacteroides.
This microbiome in traditional communities is thought to exhibit enrichment in carbohydrate- and xenobioticprocessing due to their access to more digestible sugars and therapeutic drugs (summarized in 38). In India, the gut microbiome is dominated by Firmicutes followed by Bacteroidetes, Actinobacteria and Proteobacteria and is enriched with microbial xenobiotic degradation pathways [41].

In China, the abundant taxa are Bacteroidetes, Firmicutes, Proteobacteria and Actinobacteria. However, there was a rural-urban divide with a significantly higher abundance of Proteobacteria in rural population. Urbanization was associated with loss of microbial diversity. Gene diversity increased with urbanization, along with an increase in antibiotic resistance and virulence genes, which were strongly correlated with the presence of Escherichia and Shigella [42]. In Argentina, Bacteroides was the predominant flora [43].

In all these studies, dietary habits were the main driver of microbial composition. Thus, the gut microbiomes of Kuwaitis and Argentines were similar with the predominance of Bacteroides in both populations.

The gut microbiome is thought to differ between lean and obese individuals, particularly with respect to the relative abundance of Firmicutes and Bacteroidetes [44]. In a recent cross-sectional study of nearly 4000 adult Kuwaitis, 37\% were overweight and $40.3 \%$ were obese [45]. Thus, our study participants reflected this general population, as a majority of the participants were overweight/obese. A recent systematic review noted that while several studies have identified obese individuals have a high Firmicutes: Bacteroidetes ratio compared to non-obese individuals, other studies have found contradictory results or no difference in the abundance of these phyla between obese and non-obese individuals [46]. Even though, a majority of our participants were overweight/obese, we did not observe any relationship between BMI and Firmicutes: Bacteroidetes ratio, which may be due to the small sample size. Clavel and Ecker [47] noted that a sample size of at least 500 specimens may be needed to examine associations between BMI and gut microbiome composition. Additionally, comparisons of the Firmicutes: Bacteroidetes ratio offers limited resolution given the numerous species present in these phyla.

Bacteroidetes is a complex phylum, and the class Bacteroidia is made up of five bacterial families (Bacteroidaceae, Marinilabiliaceae, Porphyromonadaceae, Prevotellaceae and Rickenellaceae), each of which is commonly found in the human gut. Bacteroides is one of the six genera under Bacteroidaceae and is a highly diverse genus, made up of numerous species (bacterio.net, List of 
Prokaryotic names with Standing in Nomenclature [35]). Bacteroides was the most prevalent and abundant genus identified in our cohort. Bacteroides spp. exist in a mutualistic relationship with the human host [48] and have an important role in fermentation of complex sugars [49], metabolism of proteins [50] and deconjugation of bile salts [51]. Bacteroidetes are enriched in patients suffering from type 1 and type 2 diabetes mellitus [52]. A recent study examining the bacterial richness of the gut microbiome of obese and lean individuals found reduced richness to be associated with adiposity, insulin resistance, dyslipidemia and an inflammatory phenotype [53]. Furthermore, participants with reduced bacterial richness had enriched Bacteroides spp. [52]. An enriched Bacteroides microbiome may be a marker of these characteristics in the Kuwaiti population.

Our phylogenetic analysis of Bacteroides revealed a high genetic diversity within individual species and suggests that species from the Bacteroides dorei/vulgatus phylogeny group may represent core gut microbiome in this Kuwaiti population. The high diversity of Bacteroides spp. across individuals has been observed in other populations [54] and may reflect differences in diets between participants; however, our small sample size prevented us from investigating this further. It is also possible that different Bacteroides ASVs present in a phylogeny group may have differing metabolic capabilities.

This study has limitations. It was difficult to recruit participants as obtaining a stool sample from a healthy person is a cultural taboo, thus the sample size was small. However, despite the small sample size, this study presents the first description of the gut microbiome composition of native Arab Kuwaiti adults. While all participants reported consuming a typical Kuwaiti diet, we did not collect detailed dietary or behavioural information leading up to specimen collection, which limits our ability to associate unique gut microbiome profiles with specific diets or behaviours. Finally, 16S rRNA gene studies are subject to primer bias, which may limit detection of relevant taxa, and provides low resolution at the species level. However, using an ASV approach provides consistent labels and allows comparison of ASVs across studies.

\section{Conclusions}

Our results showed that Firmicutes and Bacteroidetes were the dominant phyla in the gut microbiome of Arab Kuwaitis. Bacteroides was the most abundant genus with Bacteroides dorei/vulgatus forming the predominant phylogenetic group. While the predominance of Bacteroidetes may reflect the increasing Westernization of the Kuwaiti diet, larger studies examining the impact of diet and geography on the gut microbiome in the Arabian Peninsula are needed. As a majority of the study participants was overweight/obese, this also, would have contributed to the microbiome composition.

\section{Supplementary information}

Supplementary information accompanies this paper at https://doi. org/10.1186/s13099-020-00351-y.

Additional file 1: Figure S1. Alpha refraction curve of observed species Additional file 2: Table S1. The core gut microbiome is represented by 18 ASVs.

Additional file 3: Table S2. Bacteroides ASVs with average relative abundance of more than $0.50 \%$.

Additional file 4: Figure S2. Phylogeny tree showing the inferred relationship between the 225 ASVs classified in the genus Bacteroides and the $16 \mathrm{~S}$ rRNA gene sequences from the type strain for each of the recognized species in the genus Bacteroides.

Additional file 5: Table S3. Abundance and prevalence of Bacteroides phylogeny groups.

Acknowledgments

None.

Authors' contributions

MA developed the concept of the project; MA and GC performed the experiments; DB, EP and MA analysed and interpreted the results; EP, DB and MA wrote the manuscript. All authors read and approved the final manuscript.

Funding

This work did not receive specific support from any funding body.

Availability of data and materials

Sequence data are available in the NCBI Sequence Read Archive under the project access number, PRJNA554702. All other data are available in the manuscript.

\section{Ethics approval and consent to participate}

Ethical approval for the study was obtained from the Ethics Review Committee of the Health Sciences Center, Kuwait University (approval number VDR/ EC/3395). Informed written consent for stool collection was obtained from all participants.

Consent for publication

Not applicable.

Competing interests

The authors declare that they have no competing interests.

\section{Author details}

${ }^{1}$ Department of Molecular Microbiology, Murdoch Children's Research Institute, Melbourne, VIC, Australia. ${ }^{2}$ Microbiological Diagnostic Unit Public Health Laboratory, The Peter Doherty Institute for Infection and Immunity, The University of Melbourne, Melbourne, VIC, Australia. ${ }^{3}$ Melbourne Bioinformatics, The University of Melbourne, Carlton, VIC, Australia. ${ }^{4}$ Department of Microbiology, Faculty of Medicine, Kuwait University, Jabriya, Kuwait.

Received: 21 September 2019 Accepted: 15 February 2020

Published online: 26 February 2020 


\section{References}

1. Gill SR, Pop M, Deboy RT, Eckburg PB, Turnbaugh PJ, Samuel BS, et al. Metagenomic analysis of the human distal gut microbiome. Science. 2006;312(5778):1355-9.

2. El Kaoutari A, Armougom F, Gordon Jl, Raoult D, Henrissat B. The abundance and variety of carbohydrate-active enzymes in the human gut microbiome. Nat Rev Microbiol. 2013;11(7):497-504.

3. Koppel N, Maini Rekdal V, Balskus EP. Chemical transformation of xenobiotics by the human gut microbiome. Science. 2017:356(6344):eaag2770

4. Mikov M. The metabolism of drugs by the gut microflora. Europ J Drug Metab Pharmacokinet. 1994;19:201-7.

5. Biesalski HK. Nutrition meets the microbiome: micronutrients and the microbiome. Ann N Y Acad Sci. 2016;1372(1):53-64

6. O'Mahony SM, Clarke G, Borre YE, Dinan TG, Cryan JF. Serotonin, tryptophan metabolism and the brain-gut-microbiome axis. Behav Brain Res. 2015:277:32-48

7. Soto M, Herzog C, Pacheco JA, Fujisaka S, Bullock K, Clish CB, et al. Gut microbiome modulate neurobehavior through changes in brain insulin sensitivity and metabolism. Mol Psychiatry. 2018;23(12):2289-301.

8. Chung H, Pamp SJ, Hill JA, Surana NK, Edelman SM, Troy EB, et al. Gut immune maturation depends on colonization with a host-specific microbiome. Cell. 2012;149(7):1578-93.

9. Bull MJ, Plummer NT. Part 1: The human gut microbiome in health and disease. Integr Med. 2014;13(6):17-22.

10. Sekirov I, Russell SL, Antunes LC, Finlay BB. Gut microbiome in health and disease. Physiol Rev. 2010;90(3):859-904

11. Zhang J, Guo Z, Xue Z, Sun Z, Zhang M, Wang L, et al. A phylo-functional core of gut microbiome in healthy young Chinese cohorts across lifestyles, geography and ethnicities. ISME J. 2015;9(9):1979-90.

12. Yatsunenko T, Rey FE, Manary MJ, Trehan I, Dominguez-Bello MG, Contreras $\mathrm{M}$, et al. Human gut microbiome viewed across age and geography. Nature. 2012;486(7402):222-7.

13. Kurilshikov A, Wijmenga C, Fu J, Zhernakova A. Host genetics and gut microbiome: challenges and perspectives. Trends Immunol. 2017;38(9):633-47.

14. Wu GD, Chen J, Hoffmann C, Bittinger K, Chen YY, Keilbaugh SA, et al. Linking long-term dietary patterns with gut microbial enterotypes. Science. 2011:334(6052):105-8.

15. Arumugam M, Raes J, Pelletier E, Le Paslier D, Yamada T, Mende DR, et al. Enterotypes of the human gut microbiome. Nature. 2011;473(7346):174-80

16. Clemente JC, Pehrsson EC, Blaser MJ, Sandhu K, Gao Z, Wang B, et al. The microbiome of uncontacted Amerindians. Sci Adv. 2015;1 (3):e1500183.

17. Falony G, Joossens M, Vieira-Silva S, Wang J, Darzi Y, Faust K, et al. Populationlevel analysis of gut microbiome variation. Science. 2016;352(6285):560-4.

18. Brooks AW, Priya S, Blekhman R, Bordenstein SR. Gut microbiota diversity across ethnicities in the United States. PLOS Biol. 2018;16(12):e2006842.

19. Jha AR, Davenport ER, Gautam Y, Bhandari D, Tandukar S, Ng KM, et al. Gut microbiome transition across a lifestyle gradient in Himalya. PLOS Biol. 2018;16(11):e2005396

20. Zhang W, Li J, Lu S, Han N, Miao J, Zhang T, et al. Gut microbiota community characteristics and disease-related microorganism pattern in a population of healthy Chinese people. Sci Rep. 2019:9:1594

21. Dhakan DB, Maji A, Sharma AK, Saxena R, Pulikkan J, Grace T, et al. The unique composition of Indian gut microbiome, gene catalogue, and associated fecal metabolome deciphered using multi-omics approaches. GigaScience. 2019;8(3):1-20.

22. Martinez I, Stegen JC, Maldonado-Gomez MX, Eren AM, Siba PM, Greenhill AR, et al. The gut microbiota of rural Papua New Guineans: composition, diversity patterns, and ecological processes. Cell Rep. 2015;11(4):527-38.

23. Angelakis E, Yasir M, Bachar D, Azhar El, Lagier JC, Bibi F, et al. Gut microbiome and dietary patterns in different Saudi populations and monkeys. Sci Rep. 2016:6:32191.

24. Yasir M, Angelakis E, Bibi F, Azhar El, Bachar D, Lagier JC, et al. Comparison of the gut microbiome of people in France and Saudi Arabia. Nutr Diabetes. 2015:5:e153.

25. Hajjej A, Almawi WY, Arnaiz-Villena A, Hattab L, Hmida S. The genetic heterogeneity of Arab populations as inferred from HLA genes. PLOS ONE. 2018;13(3):e0192269.
26. Musaiger AO, Takruri HR, Hassan AS, Abu-Tarboush H. Food-based dietary guidelines for the Arab Gulf Countries. J Nutr Metabol. 2012;2012:905303.

27. Obesity: preventing and managing the global epidemic. Report of a WHO consultation. World Health Organ Tech Rep Ser. 2000;894: i-xii, 1-253.

28. Dehghan M, Al Hamad N, Yusufali A, Nusrath F, Yusuf S, Merchant AT. Development of a semi-quantitative food frequency questionnaire for use in United Arab Emirates and Kuwait based on local foods. Nutr J. 2005;4:18.

29. Knudsen BE, Bergmark L, Munk P, Lukjancenko O, Prieme A, Aarestrup FM, et al. Impact of sample type and DNA isolation procedure on genomic inference of microbiome composition. mSystems. 2016;1(5):e00095-16.

30. Sinclair L, Osman OA, Bertilsson S, Eiler A. Microbial community composition and diversity via 165 rRNA gene amplicons: evaluating the illumina platform. PLoS ONE. 2015;10(2):e0116955.

31. Schmieder R, Lim YW, Rohwer F, Edwards R. TagCleaner: Identification and removal of tag sequences from genomic and metagenomic datasets. BMC Bioinform. 2010;11:341

32. Callahan BJ, McMurdie PJ, Rosen MJ, Han AW, Johnson AJ, Holmes SP. DADA2: High-resolution sample inference from Illumina amplicon data. Nat Methods. 2016:13(7):581-3.

33. Oksanen J, Blanchet FG, Friendly M, Kindt R, Legendre P, McGlinn D, et al. vegan: Community ecology package. R package version 2.4-6 ed2018.

34. Warnes GR, Bolker B, Bonebakker L, Gentleman R, Liaw WHA, Lumley T, et al. gplots: various $\mathrm{R}$ programming tools for plotting data. $\mathrm{R}$ package version 3.0.1 ed2016.

35. Parte AC. LPSN - list of prokaryotic names with standing in nomenclature (bacterionet), 20 years on. Int J Syst Evol Microbiol. 2018;68(6):1825-9.

36. Sievers F, Higgins DG. Clustal Omega, accurate alignment of very large numbers of sequences. Methods Mol Biol. 2014;1079:105-16.

37. Human Microbiome Project C. Structure. function and diversity of the healthy human microbiome. Nature. 2012:486(7402):207-14.

38. Gupta VK, Paul S, Dutta C. Geography, Ethnicity or subsistence-specific variations in human microbiome composition and diversity. Front Microbiol. 2017;8:1162.

39. Shaban L, Alkazemi D. Trends in fast-food consumption among Kuwaiti youth. Int J Prev Med. 2019;10:44.

40. David LA, Maurice CF, Carmody RN, Gootenberg DB, Button JE, Wolfe BE, et al. Diet rapidly and reproducibly alters the human gut microbiome. Nature. 2014;505(7484):559-63.

41. Das B, Ghosh TS, Kedia S, Rampal R, Saxena S, Bag S, et al. Analysis of gut microbiome of rural and 'urban healthy Indians living in sea level and high altitude areas. Sci Rep. 2018:8:10104.

42. Winglee K, Howard AG, Sha W, Gharaibeh R, Liu J, Jin D, et al. Recent urbanization in China is correlated with a Westernized microbiome encoding increased virulence and antibiotic resistance genes. Microbiome. 2017:5:121

43. Carbonetto B, Fabbro MC, Sciara M, Seravalle A, Mejico G, Revasle S, et al. Human microbiota of theArgentine population - a pilot study. Front Microbiol. 2016;7:51.

44. Baothman OA, Zamzami MA, Taher I, Abubaker J, Abu-Farha M. The role of gut microbiome in the development of obesity and diabetes. Lipids Health Dis. 2016;15:108

45. Weiderpass E, Botteri E, Longenecker JC, Alkandari A, Al-Wotayan R, A Duwairi Q, et al. The prevalence of overweight and obesity in an adult Kuwaiti population in 2014. Front Endocrinol. 2019:10:449.

46. Castaner O, Goday A, Park YM, Lee SH, Magkos F, Shiow STE, et al. The gut microbiome profile in obesity: A systematic review. Int J Endocrinol. 2018:2018:4095789.

47. Clavel T, Ecker J. Microbiome and diseases: metabolic disorders. In: Haller D, editor. The gut microbiome in health and disease: Springer, Cham; 2018

48. Faith JJ, Guruge JL, Charbonneau M, Subramanian S, Seedorf H, Goodman $\mathrm{AL}$, et al. The long-term stability of the human gut microbiome. Science. 2013;341(6141):1237439.

49. Martens EC, Lowe EC, Chiang H, Pudlo NA, Wu M, McNulty NP, et al. Recognition and degradation of plant cell wall polysaccharides by two human gut symbionts. PLoS Biol. 2011:9(12):e1001221.

50. Macfarlane GT, Allison C, Gibson SA, Cummings JH. Contribution of the microflora to proteolysis in the human large intestine. J Appl Bacteriol. 1988:64(1):37-46. 
51. Narushima S, Itoha K, Miyamoto Y, Park SH, Nagata K, Kuruma K, et al. Deoxycholic acid formation in gnotobiotic mice associated with human intestinal bacteria. Lipids. 2006;41(9):835-43.

52. Larsen N, Vogensen FK, van den Berg FW, Nielsen DS, Andreasen AS, Pedersen BK, et al. Gut microbiome in human adults with type 2 diabetes differs from non-diabetic adults. PLoS ONE. 2010;5(2):e9085.

53. Le Chatelier E, Nielsen T, Qin J, Prifti E, Hildebrand F, Falony G, et al. Richness of human gut microbiome correlates with metabolic markers. Nature. 2013;500(7464):541-6.
54. Li M, Zhou H, Hua W, Wang B, Wang S, Zhao G, et al. Molecular diversity of Bacteroides spp. in human fecal microbiome as determined by group-specific 16S rRNA gene clone library analysis. Syst Appl Microbiol. 2009;32(3):193-200.

\section{Publisher's Note}

Springer Nature remains neutral with regard to jurisdictional claims in published maps and institutional affiliations.
Ready to submit your research? Choose BMC and benefit from:

- fast, convenient online submission

- thorough peer review by experienced researchers in your field

- rapid publication on acceptance

- support for research data, including large and complex data types

- gold Open Access which fosters wider collaboration and increased citations

- maximum visibility for your research: over $100 \mathrm{M}$ website views per year

At BMC, research is always in progress.

Learn more biomedcentral.com/submissions 